\title{
Feature Subset Selection Using an Optimized Hill Climbing Algorithm for Handwritten Character Recognition
}

\author{
Carlos M. Nunes ${ }^{1}$, Alceu de S. Britto Jr. ${ }^{1,2}$, \\ Celso A.A. Kaestner ${ }^{1}$, and Robert Sabourin ${ }^{3}$ \\ ${ }^{1}$ Pontifícia Universidade Católica do Paraná (PUC-PR) \\ R. Imaculada Conceição, 1155 - Curitiba (PR) 80215-901, Brazil \\ \{carlosmn, alceu,kaestner\}@ppgia.pucpr.br \\ ${ }^{2}$ Universidade Estadual de Ponta Grossa (UEPG) \\ Pç. Santos Andrade S/N, Centro - Ponta Grossa (PR) 84100-000, Brazil \\ ${ }^{3}$ École de Technologie Supérieure (ETS) \\ 1100 Rue Notre Dame Ouest - Montreal (QC) H3C 1K3, Canada \\ robert.sabourin@etsmtl.ca
}

\begin{abstract}
This paper presents an optimized Hill Climbing algorithm to select a subset of features for handwritten character recognition. The search is conducted taking into account a random mutation strategy and the initial relevance of each feature in the recognition process. The experiments have shown a reduction in the original number of features used in an MLP-based character recognizer from 132 to 77 features (reduction of $42 \%$ ) without a significant loss in terms of recognition rates, which are $99 \%$ for 60,089 samples of digits, and $93 \%$ for 11,941 uppercase characters, both handwritten samples from the NIST SD19 database. The proposed method has shown to be an interesting strategy to implement a wrapper approach without the need of complex and expensive hardware architectures.
\end{abstract}

\section{Introduction}

Many feature subset selection algorithms [1,2,3] have been developed in the last years, since this procedure can reduce not only the cost of recognition by reducing the number of features that need to be used, but in some cases it can also provide better classification accuracy. Usually, the methods found in the literature [4,5] can be categorized as: Filter or Wrapper-based approach.

In both categories, given a set of candidate features, the objective is to select a subset that performs the best under some classification system. The main difference between them is that in the Filter-based approach the relevance of each feature is defined taking into account statistical information calculated from the training dataset, while in the Wrapper approach [6,7] the classifier is used to evaluate the relevance of each feature during the selection process.

An interesting wrapper-based method was proposed in $[8,9]$ using a genetic algorithm to the subset selection. The authors have achieved a significant feature reduc- 
tion (from 132 to 100 features), which means about $25 \%$ keeping the initial classification rate almost the same $(99.16 \%)$ for handwritten digits available in the NIST SD19 database. However, their process must to evaluate 128,000 feature subset candidates. For this purpose, the authors use a cluster of 17 personal computers (with $1.1 \mathrm{GHz}$ and $512 \mathrm{Mb}$ RAM each).

In order to provide a low-cost solution in terms of architecture needed, this paper presents an optimized Hill Climbing algorithm to select a subset of features for handwritten character recognition. The search is done taking into account a random mutation strategy and a priority associated to each feature by considering its relevance in the recognition accuracy. Different from the method proposed in [8], the proposed method has shown to be an interesting strategy to implement a wrapper approach, which can be executed in more simple hardware architecture. The results presented in this paper are based on 16,000 features subset candidates selected by the proposed algorithm in a single PC $(1.3 \mathrm{GHz})$.

\section{Proposed Method}

Figure 1 presents an overview of the proposed method, in which is possible to observe 5 stages. The first stage consists of a feature extraction process, which generates 5 files (train, $\mathrm{val}_{1}, \mathrm{val}_{2}, \mathrm{val}_{3}$ and test) containing feature vectors extracted from character images.

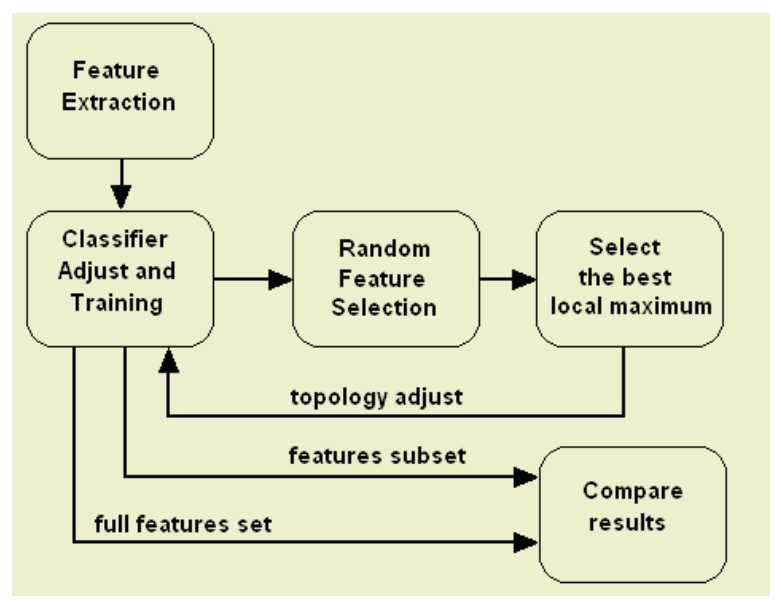

Fig. 1. Overview of the proposed method

In the second stage, a MLP neural network is trained and evaluated considering the original configuration of the feature vector and using train and $v l_{l}$. In the third stage, an optimized Hill Climbing (OpHC) algorithm uses $v l_{2}$ in order to select multiple candidates of feature subsets. A feature randomly selected is removed, or not, taking into account its relevance on the classification accuracy. In addition, a priority is associated to each feature to guide the search. 


\subsection{Feature Extraction}

The same feature extraction method described in [8] is used. It divides a character image into 6 regions and calculates 132 features based on contour, concavity and surface information.

\subsection{Classifier}

The classifier is an MLP neural network, which was used to allow the comparison with the results reported in [8,9]. The initial topology consists of 132 nodes in the input layer, 100 nodes in the hidden layer, and the output layer contains 10 or 26 nodes for digit or uppercase character recognition, respectively.

\subsection{Optimized Hill Climbing Algorithm (OpHC)}

This module consists of a modified Hill Climbing algorithm. Figure 2 shows, in italic style, the main differences of the proposed algorithm from the Original Hill Climbing (OrHC).

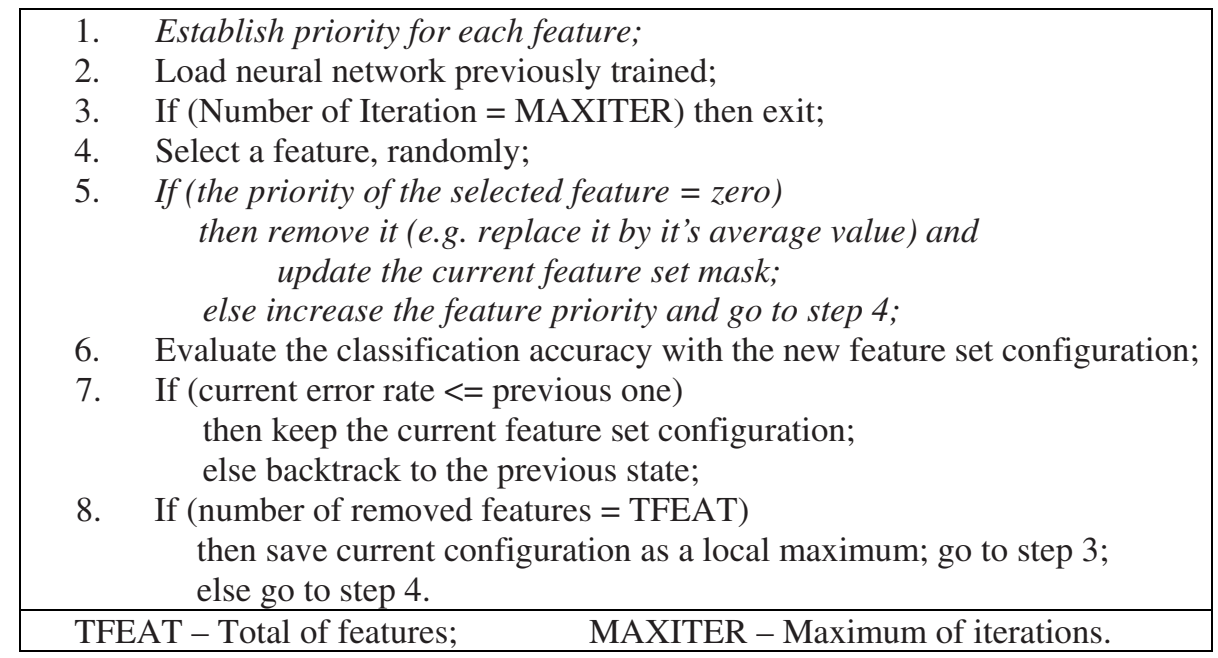

Fig. 2. Optimized Hill Climbing Algorithm (OpHC)

The algorithm starts by defining a priority for each feature (step 1) available on the initial feature vector configuration (132 for this problem). Each feature, or seed, has its priority level calculated as shown in Figure 3. In the second step of the algorithm, the neural network trained using the entire feature set is loaded. In the kernel of the algorithm (step 4), a random process is used to select a feature to be removed. In case the priory related to this feature is zero (step 5), it will be removed, otherwise its priority will be increased and a different feature will be randomly selected. The step 6 provides the current error rate after removing the selected feature. A decision about to keep the current feature set configuration is taken on step 7. For this purpose, the current error rate is compared to the previous one. As we can see, a local maximum is 
found after evaluating all the features in the current state (see step 8). After that, the $\mathrm{OpHC}$ algorithm returns to the initial state instead of backtracking to the previous one. Thus, the complete configuration of the feature set is considered again and a new feature or seed (not processed yet) is selected. The objective is to investigate other areas of the search space. In addition, the priority computed for each feature provides a way of guiding the search taking into account the feature relevance, while the concept of randomized feature removal is maintained.

1. Calculate the error rate when individual features are removed (FERROR), see Figure 4;

2. Select the maximum (MAX) and minimum (MIN) FERROR over all features;

3. Select a number of levels (NLEVELS), which is the number of priority levels to be considered;

4. Calculate the range of each level using $\mathrm{R}=(\mathrm{MAX}-\mathrm{MIN}) /$ NLEVELS;

5. The relevance (priority) of each feature is calculated as:

$\mathrm{P}=(-$ NLEVELS + (MAX-FERROR) / R $)$.

Fig. 3. Scheme to calculate the feature priority

As described before, the feature priority takes values between 0 and -NLEVELS. According to Figure 3, the highest error values receive -NLEVEL and the lowest error values receive zero. Each time that a feature is selected to removing, the algorithm evaluates its priority and in case of the priority is 0 (zero) the feature will be removed, otherwise the feature is not removed and the priority is updated $(\mathrm{P}=\mathrm{P}+1)$. Thus, when the priority reaches the value zero, the corresponding feature is removed. Figure 4 shows the initial individual feature error.

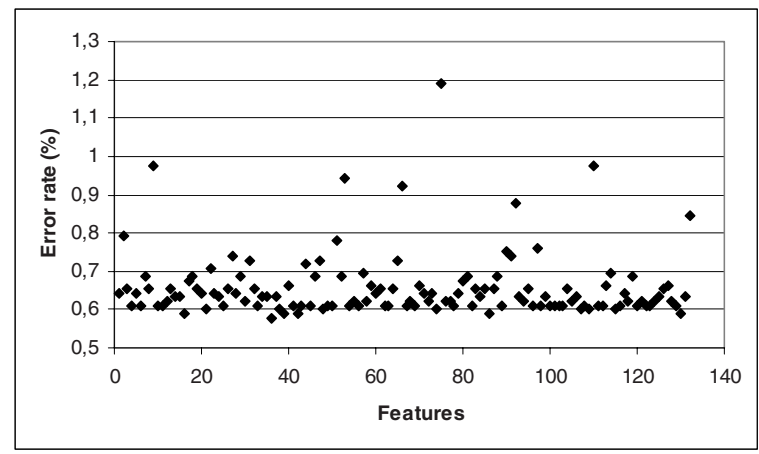

Fig. 4. Error rate after individual removal

The number of levels (NLEVELS) was experimentally defined. After evaluating the values $0,5,10,20$ and 50, the best results were obtained by using the value 10 . Figure 5 shows that, after 120 iterations the algorithm had already removed 40 features from the original set.

Another important characteristic of the proposed algorithm is the use of sensitivity analysis [8], since to retrain the neural network at each new feature subset is not fea- 


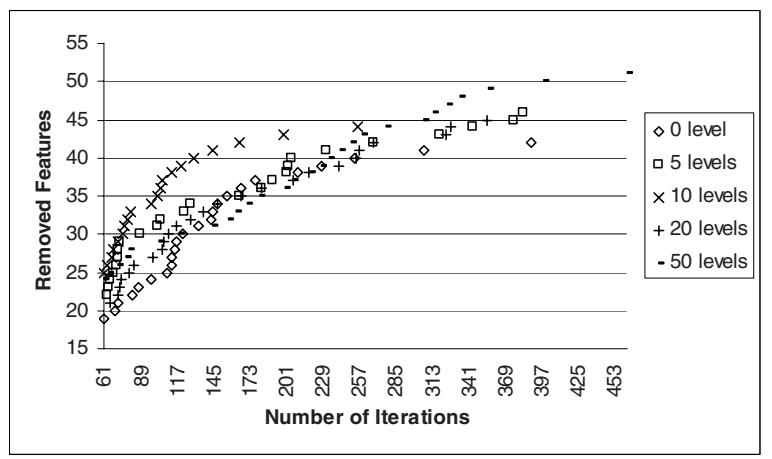

Fig. 5. Selection of the number of priority levels

sible due to the limits imposed by the learning time of the huge training set considered in this work. The sensitivity of the network is used to estimate the relationship between the input features and the network performance. So, in order to evaluate a given feature subset we replace the unselected features by their averages values (step 5 Figure 3) evaluated on the training database. In this way, we avoid training the neural network and hence turn the wrapper approach feasible for our problem.

Each local maximum found by this module represents a feature subset candidate. The search for feature subsets is done on $\mathrm{val}_{2}$.

\subsection{Selection of the Best Feature Subset Candidate}

A different validation dataset $\left(\mathrm{Nal}_{3}\right)$ is used to select one feature subset from those candidates provided by the OpHC algorithm. The selection is done based on the recognition accuracy. Another strategy used to select the best feature set configuration is selecting that with represents the smallest feature subset - also evaluated in our experiments.

\subsection{Final Evaluation}

In this module, the final feature subset selected in the last stage is used to retrain the neural network, whose topology is adapted to this new configuration of the feature set. A final evaluation is done using the test file.

\section{Experimental Results and Discussion}

The experiments are based on handwritten digits and uppercase characters available in the NIST SD19 database. In the experiment based on digits the following protocol was used: 195,000 samples for training (train) and 28008 for validation (9,336 in $v a l_{1}, 9,336$ in $v a l_{2}$ and 9,336 samples in $v l_{3}$ ) - all samples from series hsf_0, 1, 2 and 3 series. Other 60,089 samples were used for testing (test), which are available on hsf_7 series. 
The data sets used in the experiments using uppercase characters are composed of 37,440 samples for training (train) and 12,092 for validation (4,031 in $\mathrm{val}_{1}, 4,031$ in $v a l_{2}$ and 4,030 in $v a l_{3}$ ) - all samples from hsf_0,1,2 and 3 series. Other 11,941 samples from hsf_4 were used for testing (test).

In the experimental protocol, $\mathrm{val}_{1}$ is used during training of the neural network to avoid an overtraining. $\mathrm{Val}_{2}$ is used during the search for feature subset candidates performed by the $\mathrm{HC}$ algorithm. Finally, $\mathrm{val}_{3}$ is used to select the subset of features, which provides the best recognition accuracy among the candidates provided by the $\mathrm{HC}$ algorithm. The testing set (test) is used as a black box just to compare the final recognition results of the classifier after his topology has been adapted to the new configuration of the feature set.

\subsection{Experiments on Handwritten Digits}

Both algorithms, the original and optimized HC were evaluated. The MAXITER variable was set to 16,000 iterations or solutions. At the end of the iterations the original HC has used only 3 seeds, since it does not returns to the initial configuration of the feature set (initial state) after finding a local maximum or after removing all features in the current state, but it returns to the previous state. From this three seeds or starting points, the original algorithm found 172 local maximum (subset candidates). By contrast, the modified algorithm has investigated with the priority scheme all possible seeds (132) using the same 16,000 iterations and it has generated 47 feature subset candidates. In fact, the proposed algorithm has stopped after all features have been used as seed. This means that all features were removed during the search and a bigger diversity on evaluated solutions was reached.

In Figure 6, each point represents a feature subset candidate of the 172 found by using the Original Hill Climbing (OrHC). As we can observe, all points are very concentrated in a small area of the search space. By contrast, in Figure 7, the 47 feature subset candidates found by using the modified HC are not concentrated as those provided by the original algorithm. The reason is that in the modified algorithm each time a local maximum is found, the initial configuration of the feature set is returned in order to select a new seed. Moreover, the strategy of using the priority scheme has provided to the algorithm a faster convergence to a local maximum.

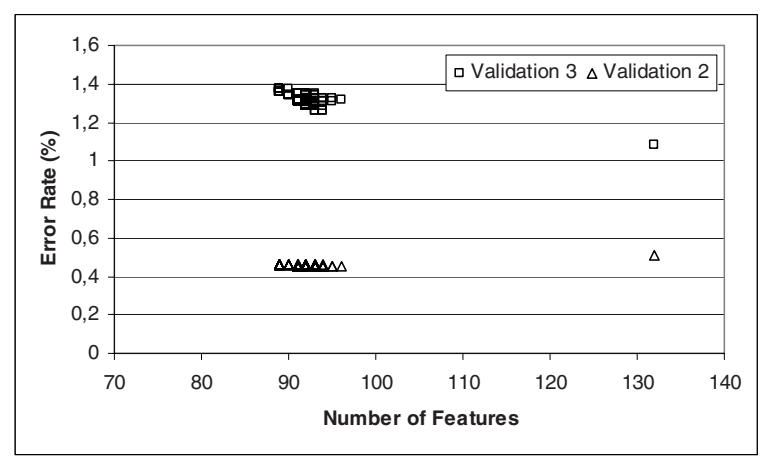

Fig. 6. Feature subset candidates provided by the traditional $\mathrm{HC}$ algorithm 


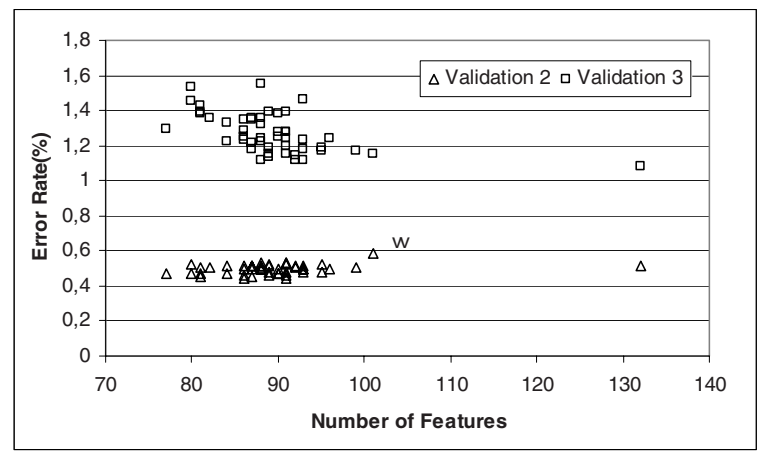

Fig. 7. Feature subset candidates provided by the optimized $\mathrm{HC}$ algorithm

In a first set of experiments, the $\mathrm{val}_{3}$ dataset is used to evaluate and select the best local maximum (or feature subset) from the 172 provided by the original HC algorithm (OrHC). The same strategy is used to select a local maximum from the 47 provided by the optimized algorithm $(\mathrm{OpHC})$. In addition, we also experiment to consider just the number of features removed, since the classifier has shown a small loss in terms of accuracy. This means that, in this experiment, the best configuration corresponds to the smallest subset of features.

As we can observe in Table 1, there is no significant loss in terms of classification accuracy. However, it is possible to observe a reduction of features when the optimized HC algorithm was used.

Table 1. Experimental results on digits

\begin{tabular}{lrrr}
\hline Experiment & \# Features & Training & Testing \\
& & & \\
\hline Initial configuration of the feature set & 132 & $99.77 \%$ & $99.10 \%$ \\
OrHC (using val $_{3}$ ) & 92 & $99.50 \%$ & $99.04 \%$ \\
OpHC (using val ${ }_{3}$ ) & 87 & $99.95 \%$ & $98.95 \%$ \\
OrHC (smallest feature set) & 81 & $99.50 \%$ & $98.92 \%$ \\
OpHC (smallest feature set) & 77 & $99.86 \%$ & $98.94 \%$ \\
\hline
\end{tabular}

In order to compare our results with those obtained by Soares [8], we have used the same experimental protocol. The proposed wrapper approach has shown a more significant reduction $(42 \%)$ than that observed by using GA $(25 \%)$. Moreover, while the wrapper-based approach proposed by Soares [9] needs to evaluate around 128,000 solutions running in a cluster with 17 machines, the proposed method evaluates just 16,000 solutions using a Pentium III (1.3 GHz).

\subsection{Experiments on Handwritten Characters}

The method was also applied to handwritten uppercase characters available in the NIST SD19 database. During the experiments, just the optimized HC was used. However, the experiments considers to select the final feature set configuration taking into 
Table 2. Experimental results on uppercase characters

\begin{tabular}{lrrr}
\hline Experiment & \# Features & Training & Testing \\
& & & \\
\hline Initial configuration of the feature set & 132 & $97.23 \%$ & $93.05 \%$ \\
OpHC (validation3) & 101 & $97.57 \%$ & $92.51 \%$ \\
OpHC (smallest feature set) & 79 & $97.68 \%$ & $92.50 \%$ \\
\hline
\end{tabular}

account the recognition rate on $\mathrm{val}_{3}$ dataset, and also it considers the more significant reduction on the number of features.

The reduction of feature was about $24 \%$ in the first experiment and about $40 \%$ in the last one.

\section{Conclusion}

The proposed method has shown to be an interesting strategy to implement a wrapperbased method, which can be executed in low-cost hardware architecture. In the experiments, a reduction in the original number of features 132 to 77 features (around $42 \%$ ) without a significant loss in terms of digit recognition rate was observed. Similar result was observed for uppercase characters, reduction around $40 \%$.

\section{References}

1. Jain, A., Duin, R. P. W., Mao, J.: Statistical Pattern Recognition: A Review. IEEE Transactions on Pattern Analysis and Machine Intelligence, Vol. 22(1). (2000) 4-37

2. Boz, O.: Feature Subset Selection by Using Sorted Feature Relevance. ICMLA International Conference on Machine Learning and Application, Las Vegas City, (2002)

3. Molina, L. C., Belanche, L., Nebot, À.: Feature Selection Algorithms: Survey Experimental and Evaluation. IEEE International Conference on Data Mining, Vol. 1. Maebashi City (2002) 306-313

4. Blum, A. L., Langlev P.: Selection of Relevant Features and Examples in Machine Learning. special issue Artificial Intelligence, Vol. 97. N.1-2 (1997) 245-271

5. Aha, D. W., Bankert, R. L.: Feature Selection for Case-based Classification of Cloud Types: an empirical comparison. Working Notes of the AAAI, Workshop on Case-Based Reasoning, Vol. 1. (1994) 106-112

6. Raman, B., Ioerger, T. R.: Enhancing Learning Using Feature and Example Selection. Journal of Machine Learning Research, submitted for publication, (2003)

7. Kohavi, Ron, John, George H., Wrappers for Feature Subset Selection. in Artificial Intelligence Journal, Special Issue On Relevance, Vol. 97. N. 1 (1997) 273-324

8. Oliveira, L. S., Sabourin, R., Bortolozzi, F., Suen, C. Y.: Feature Selection Using Multiobjective Genetic Algorithms for Handwritten Digit Recognition. Proc. International Conference on Pattern Recognition, Vol. 1. Quebec City (2002) 568-571

9. Oliveira, L.S., Sabourin, R., Bortolozzi, F., Suen, C.Y.: A Methodology for Feature Selection Using Multi-objective Genetic Algorithms for Handwritten Digit String Recognition. in the International Journal of Pattern Recognition and Artificial Intelligence (IJPRAI), Vol. 17. N. 6 (2003) 903-930 